\title{
Diffusion weighted magnetic resonance imaging of compromised tissue in stroke
}

Institute of Child Health, University College London Medical School, London : Radiology and Physics Unit

A Connelly

D G Gadian

Neurosciences Unit V Ganesan

F J Kirkham

Great Ormond Street Hospital for Children NHS Trust, London: Department of Radiology

W K Chong

C L Johnson

Correspondence to: Dr Alan Connelly, MR2, Department of Radiology, Great Ormond Street Hospital for Children, London WC1N 3JH.

Accepted 23 April 1997

\author{
A Connelly, W K Chong, C L Johnson, V Ganesan, D G Gadian, F J Kirkham
}

\begin{abstract}
Diffusion weighted imaging (DWI) and T2 weighted magnetic resonance imaging were performed on at least two occasions in 28 children presenting with stroke. In previous reports of DWI in human stroke, eventual infarction was observed (with only one exception) in all regions in which early DWI hyperintensity occurred. In the present report, two children had regions of DWI hyperintensity which did not progress to infarction. One patient who presented with right hemiplegia showed extensive high signal on DWI, with T2 evidence of tissue swelling but without hyperintensity. DWI changes persisted over weeks, with no imaging indication of infarction. This child recovered completely. A second child who had a major vessel infarct with concomitant regions of hyperintensity on $T 2$ weighted imaging and DWI, also had DWI hyperintensity in an adjacent territory which did not develop any subsequent evidence of infarction. Thus in clinical practice DWI can demonstrate tissue which is compromised but not irreversibly so.

(Arch Dis Child 1997;77:38-41)
\end{abstract}

Keywords: magnetic resonance imaging; diffusion weighted imaging; stroke; ischaemia

Ischaemic stroke occurs in childhood with an annual incidence of approximately 1.25 per $100000 .{ }^{1}$ The aetiology is often obscure, although conventional angiography reveals cerebrovascular disease in over $80 \%$ of cases. ${ }^{2}$ Functional recovery often appears good, particularly in young children, but dense hemiplegia may occur and there is a significant mortality. ${ }^{3}$

Early diagnosis of the presence and extent of cerebral damage will become increasingly important if treatment becomes available, particularly as any therapeutic window is likely to be narrow. Within the first few hours after stroke, both computed tomography and conventional magnetic resonance imaging (MRI) are insensitive in the detection of infarcts. ${ }^{4}$ The relation between the imaging findings and the clinical syndromes of transient ischaemic attack (resolution of symptoms and signs within 24 hours), reversible ischaemic neurological deficit (resolution beyond 24 hours), and completed stroke (permanent neurological deficit) remains unclear, since infarction may be demonstrated in patients who make a full recovery within 24 hours $^{4}$ and follow up MRI may be negative in patients with persisting symptoms and signs. ${ }^{5}$ However, further information should become available through the use of diffusion weighted imaging (DWI), a magnetic resonance technique that is much more sensitive than more conventional MRI sequences to acute events in ischaemia ${ }^{6}$; animal models show that affected regions can be visualised by DWI within minutes of an ischaemic insult (see ${ }^{8}$ for review).

In diffusion weighted imaging, signal attenuation is seen in regions with free diffusion of water, with a resultant relative signal hyperintensity in regions of restricted diffusion and parallel reduction in the apparent diffusion coefficient in brain tissue. The decrease in diffusion of water observed in early ischaemia is believed to reflect the shift of water from extracellular to intracellular compartments. ${ }^{8}$ There is evidence that this process of cell swelling is related to failure of the energy dependent ion pumps and the breakdown of membrane potential. ${ }^{9}$ However, observations in an animal model indicate that such redistribution of water (and DWI hyperintensity) can occur in some regions initially in the absence of energy failure, and is associated more closely at that stage with tissue acidosis than with loss of ATP. ${ }^{10}$

An important concept in the search for treatments in stroke is that of the ischaemic penumbra, a term used to describe the region of ischaemic tissue peripheral to the core of an infarct in which the blood flow reduction has been sufficient to compromise the tissue, but in which cells may remain viable. The existence of the penumbra in humans has been questioned, because it has not been possible to visualise it previously or to describe its temporal evolution to infarction. However, sequential MRI studies using DWI and T2 weighted imaging may now allow investigation of the penumbra in humans, including the degree to which the cellular changes reflected by DWI may be reversible. Animal models ${ }^{11}{ }^{12}$ suggest that DWI may be able to detect reversible changes as well as those that inevitably progress to infarction.

From a series of children with neurological presentation of stroke in whom DWI and T2 weighted examinations were performed, we report the observation of DWI hyperintensity - in one case persisting over at least 10 days - in brain regions which evidently do not progress to infarction. 

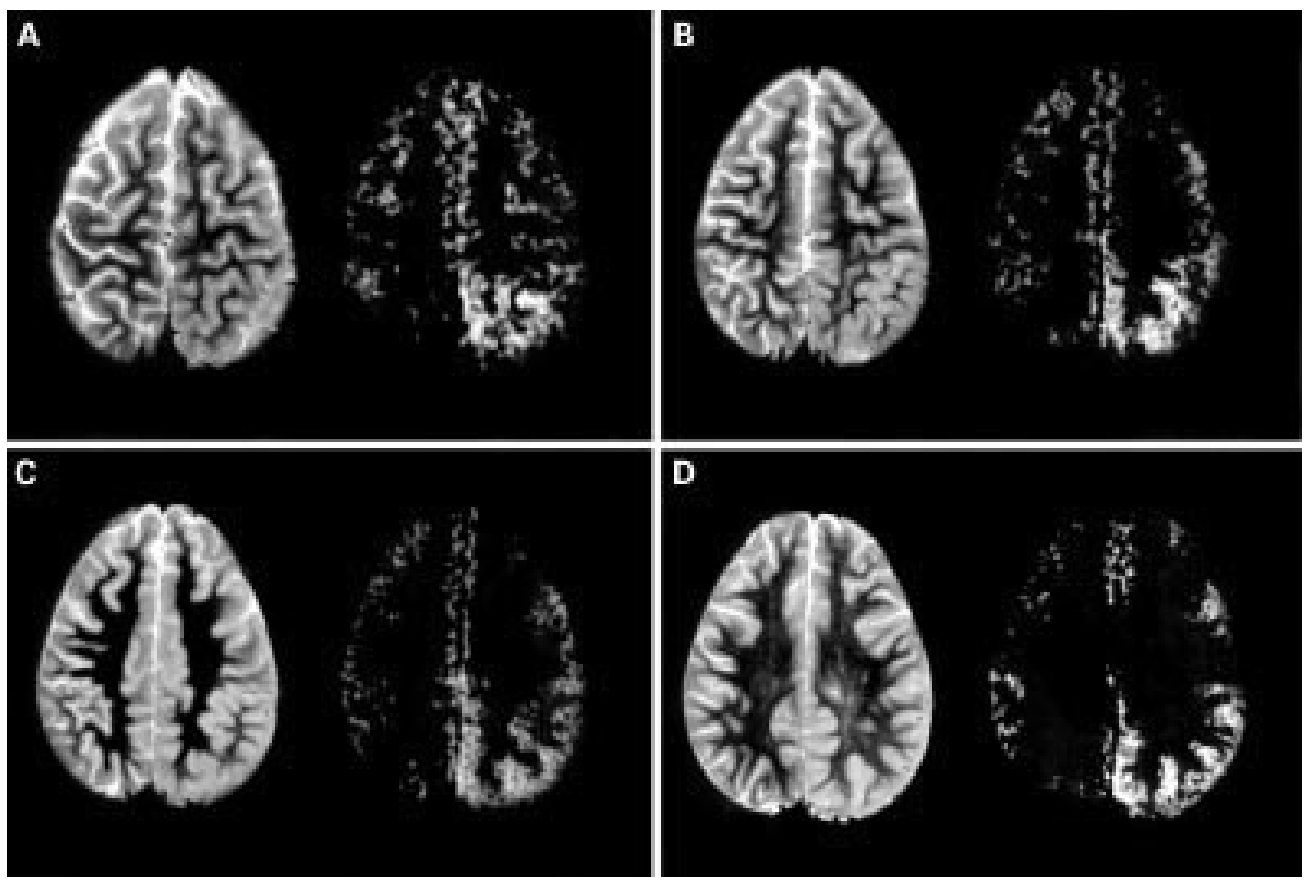

Figure 1 Axial T2 weighted images (left hand image in each pair) and diffusion weighted images (right hand image in each pair) of patient 1 acquired 10 days after his initial presentation. (A) to (D) show images obtained from a descending series of slice positions starting near the top of the head. In each case, the T2 weighted images show areas of swollen cortex without hyperintensity in the left parieto-occipital region (the apparent focal high signal in the white matter in the left hemisphere in (D) is due to partial volume of the upper part of the lateral ventricle, as evidenced by the lower slices). The corresponding diffusion weighted images show signal hyperintensity in the equivalent anatomical locations to the areas of tissue swelling.

\section{Methods}

PATIENTS

All children presenting to Great Ormond Street Hospital with stroke or with risk factors for cerebrovascular disease, for example sickle cell disease or moya moya, were eligible for DWI. Stroke was defined as a rapidly developed focal neurological deficit of more than 24 hours duration in order to encompass the full spectrum of aetiologies responsible for ischaemic lesions in childhood. Twenty eight children with stroke had two or more magnetic resonance investigations which included DWI; of these, 23 had the first scan within 60 hours of the onset of their symptoms. In the majority of cases, the observed progression of diffusion and T2 weighted signal intensities was consistent with that previously reported in adult patients. However, in some children this was not the case, and it is two of these latter patients who form the basis of this report.

MAGNETIC RESONANCE

All MRI examinations were performed on a standard clinical $1.5 \mathrm{~T}$ Siemens SP4000 system. The first magnetic resonance scan was performed as soon as possible after admission and a second scan was performed before discharge. As well as DWI, all children had a T2 weighted image and magnetic resonance angiography on each occasion.

T1 weighted and T2 weighted images were acquired using a double echo inversion recovery $(\mathrm{IR})$ sequence $(\mathrm{TI}=145 \mathrm{~ms}, \mathrm{TR}=4000$ $\mathrm{ms}, \mathrm{TE}=23$ and $85 \mathrm{~ms}$ ). DWI was performed using a high speed STEAM sequence, ${ }^{13}$ similar to that described previously in adult stroke studies. ${ }^{14}$ The three radiofrequency pulses used in this present work consisted of two nonselective $90^{\circ}$ pulses followed by a slice selective pulse. The image parameters were $\mathrm{TR}=16 \mathrm{~ms}$, flip angle $=12^{\circ}$, matrix $=128 \times 128$, slice thickness $=8$ or $10 \mathrm{~mm}, \mathrm{TM}=400 \mathrm{~ms}$, with a field of view of $200 \mathrm{~mm}$ or $210 \mathrm{~mm}$. The time between the two $90^{\circ}$ pulses was $11 \mathrm{~ms}$, resulting in a TE of $22 \mathrm{~ms}$. Diffusion weighting gradients were applied simultaneously in all three directions. The diffusion weighting gradient strength in each direction was $9 \mathrm{mT} / \mathrm{m}$, with a duration of $4 \mathrm{~ms}$. Ten to 15 diffusion weighted images were acquired at each slice position (with each image requiring approximately four seconds acquisition time), and these were subsequently summed to generate the diffusion weighted images shown. Data addition was done in the image domain in order to minimise potential image artefacts resulting from movement. Diffusion weighted images were acquired from nine slice positions.

\section{Results}

CLINICAL AND MAGNETIC RESONANCE

Patient 1 initially presented at the age of 2 years with a left hemiparesis, from which he made a complete recovery, associated with only slight right hemisphere atrophy and no evidence of focal infarction on MRI. Formal arteriography after this earlier episode was normal. A year later he developed an acute right hemiparesis preceded by vomiting and irritability. This more recent neurological deficit resolved completely over the following few months. An electroencephalogram showed an excess of slow activity in the hemisphere contralateral to the hemiparesis on both occasions. The patient's 


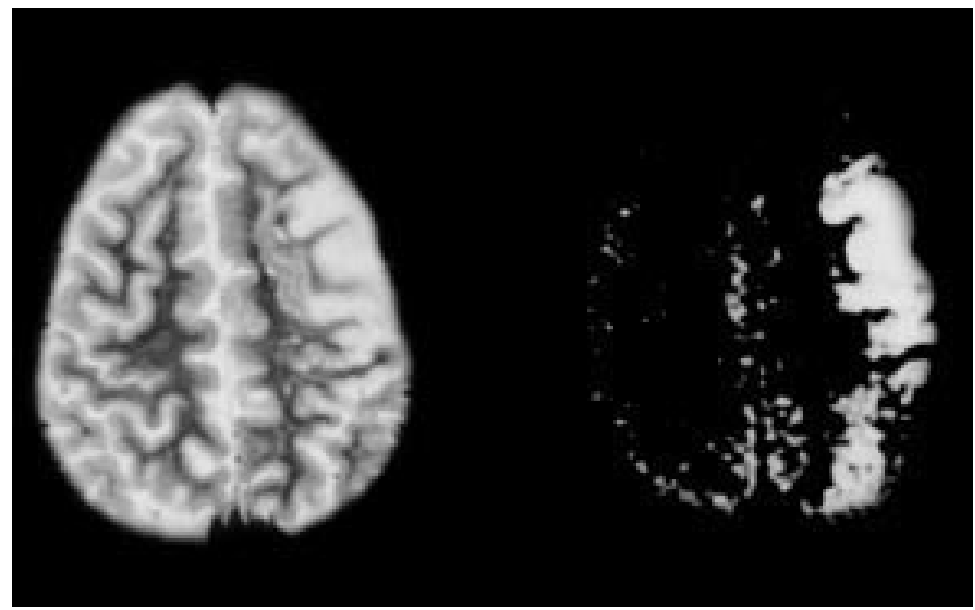

Figure 2 Axial T2 weighted (left) and diffusion weighted (right) images from patient 2 at the time of initial presentation (32 hours after onset). Both T2 weighted and diffusion weighted images show high signal regions consistent with a middle cerebral artery territory infarct. The diffusion weighted image also shows hyperintensity extending into the parietal region, but with no corresponding signal change on the T2 weighted image. T2 weighted images acquired 10 days later showed signal change consistent with infarction in the frontal area. However, at this time there was no evidence of infarction in the posterior region initially abnormal on DWI, while the DWI hyperintensity in this area was no longer apparent. DWI = diffusion weighted imaging.

mother had a history of migraine with aura and a diagnosis of hemiplegic migraine was made. The child has been on flunarizine with no recurrent episodes.

An initial magnetic resonance investigation was performed 20 hours after the onset of his more recent right hemiparesis. DWI hyperintensity was observed in the left frontal and left parieto-occipital regions, and was associated with tissue swelling but not with signal increase on $\mathrm{T} 2$ weighted or inversion recovery $\mathrm{T} 1$ weighted scans. On further magnetic resonance investigation after approximately two weeks, the parieto-occipital DWI hyperintensity had increased significantly in extent, but the $\mathrm{T} 1$ weighted and T2 weighted images still showed only tissue swelling without hyperintensity (fig 1). No evidence of an infarct was observed. Two magnetic resonance angiograms appeared normal. Throughout this two week period, the child had a marked right hemiparesis from which he subsequently made a full recovery over the following few months.

Patient 2 was a 7 year old girl with sickle cell disease (HbSS). Her first symptom was of subtle deterioration in the quality of her handwriting. Five days later, she became acutely drowsy and aphasic and went on to develop a dense right hemiparesis and right sided focal seizures. She required 48 hours of ventilatory support for airway protection and was treated with exchange transfusions. She has a residual dense right hemiparesis and has suffered significant decline in her level of cognitive functioning.

This patient was found to have middle cerebral artery occlusion on magnetic resonance angiography (corroborated by transcranial Doppler ultrasound studies). Extensive high signal in the left frontoparietal region was observed on both DWI and T2 weighted imaging on initial magnetic resonance investigation (32 hours postictal; fig 2). At the follow up scan a further 10 days later, this region showed pseudonormalisation on DWI and high signal with disruption of the cortical structure on T2 weighted imaging. This pattern of imaging changes is typical of that seen in the majority of our stroke patients. However, on the initial scan in this patient, additional DWI hyperintensity was found to extend into the ipsilateral posterior parietal lobe when this region appeared spared on T2 weighted imaging (fig 2). After 10 days, this posterior region was no longer hyperintense on DWI, while T2 weighted imaging showed intact structure without high signal, in contrast to the appearance of the large infarct throughout the remainder of the middle cerebral artery territory.

\section{Discussion}

The imaging findings in the middle cerebral artery territory infarct in patient 2 are consistent with previous data from stroke in humans. ${ }^{14-19}$ These have shown that in patients with cerebrovascular disease, regions of focal infarction (as demonstrated on T2 weighted imaging) show early DWI hyperintensity, and that, if imaged sufficiently early in the process, the DWI abnormality appears obvious when the abnormality on T2 weighted imaging is either absent or more subtle.

The data presented here also show evidence that DWI may visualise changes associated with ischaemia in regions which do not develop signs of infarction on conventional MRI. All but one patient in the above studies ${ }^{14-19}$ in whom early DWI changes were observed were reported to show evidence of infarction in corresponding locations on subsequent scans. However, in a study of 29 patients using navigated spin echo DWI, Marks et al ${ }^{19}$ reported one case of reversible ischaemic neurological deficit in whom no T2 weighted imaging abnormality was observed at any stage, but in whom DWI showed high signal on images acquired 18 hours after symptom onset. This DWI hyperintensity was no longer observed on imaging at 36 hours after the event, while the patient's symptoms had resolved at 24-30 hours.

In the patient with a clinical diagnosis of hemiplegic migraine (patient 1), DWI changes not only persisted but increased in extent over a period of 10 days. These were accompanied by evidence of tissue swelling but not by signal increase on $\mathrm{T} 2$ weighted images (fig 1), nor by signal abnormality on inversion recovery $\mathrm{T} 1$ weighted images. The motor deficit in this patient recovered completely over a period of several months. Thus DWI hyperintensity does not necessarily augur infarction, and in this case was associated only with cerebral swelling, with the tissue apparently recoverable. A similarly prolonged period of hemiparesis contralaterally which had occurred one year previously, also left no motor deficit, and there was no evidence of focal infarct related to that episode on MRI performed at the time of the later presentation. It has been reported previously that hemispheric cerebral blood flow is reduced in hemiplegic migraine, ${ }^{20}$ with the suggestion that this may be below the threshold at which membrane potential can be sustained. One 
proposed mechanism for migraine, namely spreading depression (spreading waves of depolarisation propagating at approximately 3 $\mathrm{mm} / \mathrm{min}$ ), has been shown to be demonstrable using DWI ${ }^{21}$ or $\mathrm{T}^{\star}{ }^{\star}$ weighted ${ }^{22}$ magnetic resonance techniques. However, the DWI results in this present study had no characteristics suggesting that such a mechanism was involved in this case of hemiplegic migraine.

Patient 2 had DWI abnormality initially in the posterior parietal region adjacent to the obvious infarct (fig 2) described above. This posterior region showed no further DWI or T2 signal hyperintensity on subsequent scans, and may be on the border of the middle cerebral artery territory protected by collateral supply from the posterior cerebral artery. The extent and severity of ischaemic damage to tissue is known to be dependent on both the magnitude and duration of the reduction in blood flow, ${ }^{23}$ which depends not only on the main vascular supply to the threatened tissue, but also on any available collateral. This case suggests that DWI is able to visualise not only the relatively major focal effects of a middle cerebral artery occlusion, but also the lesser degree of ischaemia associated with either a marginal collateral supply or a global reduction in cerebral perfusion pressure, the effects of which may be reversible. Whether or not there is any selective neuronal loss in these apparently spared regions may require detailed neuropathological study, although it is possible that proton magnetic resonance spectroscopy may provide useful information through the signal from $N$-acetylaspartate (NAA), which is widely regarded as a neuronal marker. This question is of great importance since it is well recognised that stroke patients who make a good recovery in terms of motor function may be left with subtle neuropsychological difficulties. A systematic approach will be needed to determine the factors predicting whether or not vascular disease leads to infarction of the whole or only part of the territory supplied by that vessel in human stroke; magnetic resonance imaging, including DWI, offers a means of approaching this important question.

In conclusion, we have shown that diffusion weighted imaging can not only demonstrate early evidence of ischaemia in regions which eventually infarct, but may also show tissue which is compromised but not irreversibly so. Further studies will be needed to determine the mechanisms underlying the reversible DWI hyperintensity, and a larger cohort of patients with parallel cerebral blood flow studies and long term outcome is required to understand the significance of these apparently reversible lesions.
This work was supported by the Wellcome Trust.

1 Broderick J, Talbot GT, Prenger E, Leach A, Brott T. Stroke in children within a major metropolitan area: the surprising importance of intracerebral hemorrhage. $f$ Child Neurol importance of in

2 Shirane R, Sato S, Yoshimoto T. Angiographic findings of ischemic stroke in children. Child Nerv Syst 1992;8:432-6.

3 Higgins JJ, Kammerman LA, Fitz CR. Predictors of survival and characteristics of childhood stroke. Neuropediatrics 1991;22:190-3.

4 Mohr JP, Biller J, Hilal SK, et al. Magnetic resonance versus computed tomographic imaging in acute stroke. Stroke 1995;26:807-12.

5 Alberts MJ, Faulstich ME, Gray L. Stroke with negative brain magnetic resonance imaging. Stroke 1992;23:663-7. 6 Moseley ME, Cohen Y, Mintorovitch J, et al. Early detection of regional cerebral ischemia in cats: comparison of diffusion- and T2-weighted MRI and spectroscopy. Magn Reson Med 1990;14:330-46.

7 Mintorovitch J, Moseley ME, Chileuitt L, Shimizu H, Cohen Y, Weinstein PR. Comparison of diffusion- and ischemia and reperfusion in rats. Magn Reson Med 1991;18: $39-50$.

8 Van Bruggen N, Roberts TP, Cremer JE. The application of magnetic resonance imaging to the study of experimental cerebral ischaemia. Cerebrovasc Brain Metab Rev 1994;6: 180-210.

9 Busza AL, Allen KL, King MD, Van Bruggen N, Williams SR, Gadian DG. Diffusion-weighted imaging studies of cerebral ischemia in gerbils. Potential relevance to energy failure. Stroke 1992;23:1602-12.

10 Kohno K, Hoehn-Berlage M, Mies G, Back T, Hossmann KA. Relationship between diffusion-weighted MR images, cerebral blood flow, and energy state in experimental brain infarction. Magn Reson Imaging 1995;13:73-80.

11 Minematsu K, Li L, Sotak CH, Davis MA, Fisher M. Reversible focal ischemic injury demonstrated by diffusionweighted magnetic resonance imaging in rats. Stroke 1992; 23:1304-10.

12 Lo EH, Matsumoto K, Pierce AR, Garrido L, Luttinger D. Pharmacologic reversal of acute changes in diffusionweighted magnetic resonance imaging in focal cerebral ischemia. F Cereb Blood Flow Metab 1994;14:597-603.

13 Merboldt KD, Hanicke W, Bruhn H, Gyngell ML, Frahm J. Diffusion imaging of the human brain in vivo using high-speed STEAM MRI. Magn Reson Med 1992;23:17992 .

14 Warach S, Chien D, Li W, Ronthal M, Edelman RR. Fast magnetic resonance diffusion-weighted imaging of acute human stroke. Neurology 1992;42:1717-23.

15 Warach S, Gaa J, Siewert B, Wielopolski P, Edelman RR. Acute human stroke studied by whole brain echo planar diffusion-weighted magnetic resonance imaging. Ann Neurol 1995;37:231-41.

16 De Crespigny AJ, Marks MP, Enzmann DR, Moseley ME. Navigated diffusion imaging of normal and ischemic havigated diffusion imaging of normal and

17 Chien D, Kwong KK, Gress DR, Buonanno FS, Buxton $\mathrm{RB}$, Rosen BR. MR diffusion imaging of cerebral infarction in humans. Am $\mathcal{F}$ Neuroradiol 1992;13:1097-102.

18 Warach S, Dashe JF, Edelman RR. Clinical outcome in ischemic stroke predicted by early diffusion-weighted and perfusion magnetic resonance imaging: a preliminary analysis. F Cereb Blood Flow Metab 1996;16:53-9.

19 Marks MP, de Crespigny AJ, Lentz D, Enzmann DR, Albers GW, Moseley ME. Acute and chronic stroke: navigated spin-echo diffusion-weighted MR imaging. Radiology 1996; 199:403-8.

20 Friberg L, Olsen TS, Roland PE, Lassen NA. Focal ischaemia caused by instability of cerebrovascular tone during attacks of hemiplegic migraine. A regional cerebral blood flow study. Brain 1987;110:917-34

21 Hasegawa Y, Latour LL, Formato JE, Sotak CH, Fisher M. Spreading waves of a reduced diffusion coefficient of water in normal and ischemic rat brain. F Cereb Blood Flow Metab 1995;15:179-87.

22 Gardner-Medwin AR, Van Bruggen N, Williams SR, Ahier RG. Magnetic resonance imaging of propagating waves of spreading depression in the anaesthetised rat. 7 Cereb Blood Flow Metab 1994;14:7-11.

23 Jones TH, Morawetz RB, Crowell RM, et al. Thresholds of focal cerebral ischemia in awake monkeys. $f$ Neurosurg 1981;54:773-82. 